\title{
Implementation of Feeder Automation Using Colored Petri Nets
}

\author{
Chia-Hung Lin, ${ }^{1 *}$ Chung-Sheng $\mathrm{Li},{ }^{1}$ Te-Tien $\mathrm{Ku},{ }^{1}$ and Chin-Ying $\mathrm{Ho}^{2}$ \\ ${ }^{1}$ Department of Electrical Engineering, National Kaohsiung University of Science and Technology, \\ 415, Jiangong Rd., Sanmin Dist., Kaohsiung City 80778, Taiwan \\ ${ }^{2}$ Dept. of Electrical Engineering, Kao Yuan University, \\ 1821 Jhongshan Rd., Lujhu Dist., Kaohsiung City 82151, Taiwan
}

(Received September 8, 2020; accepted February 2, 2021)

Keywords: feeder automation, distribution automation system, colored Petri net, remote terminal unit, feeder terminal unit

Feeder automation is one of the major functions of the distribution automation system (DAS) of Taiwan Power Company (Taipower). Feeder automation reduces the customer outage duration when a fault contingency occurs in a distribution feeder. In feeder automation operation, the feeder terminal unit (FTU) measures and monitors the voltage and current sensors of distribution line switchgear in real time. To propose the service restoration strategy for more efficient feeder automation, an inference mechanism using a colored Petri net (CPN) is employed to derive appropriate switching operations by considering the operational constraints of a distribution system. A CPN model for feeder automation that includes automatic line switches and circuit breakers is proposed for fault detection, isolation, and restoration (FDIR). To demonstrate the effectiveness of the proposed methodology, the computer simulation of a Taipower distribution system with 18 feeders is carried out. The results show that the proposed CPN model can be applied efficiently for the FDIR of the distribution system.

\section{Introduction}

Traditionally, electric utilities detect power outages of distribution systems via customer contact about outages. The distribution dispatch control center then sends a crew to visit the site of an open breaker or blown fuse, and switches are operated to isolate the fault and restore the service. Owing to rapid load growth and the delay in the construction schedule for new substations and feeders, some transformers and feeders do not have enough reserve capacity for non-interrupted load transfer. In particular, service restoration in a distribution system is difficult when a fault occurs in summer.

To solve the above problem, Taiwan Power Company (Taipower) has had an active distribution automation system (DAS) since 1991. The overcurrent relay of the feeder terminal unit (FTU) of the automatic line switch identifies the fault location when a permanent fault occurs on a primary feeder. An FTU is a smart device that detects power failures and transmits the power quality information needed for the operation of the distribution system to the central control unit. It does this by measuring, monitoring, and controlling data from the voltage and

*Corresponding author: e-mail: chlin@nkust.edu.tw https://doi.org/10.18494/SAM.2021.3165 
current sensors of distribution line $(22.8 / 11.4 \mathrm{kV})$ switchgear in real time. On the basis of the fault flags of the FTU, the master station of the DAS issues a command to open the boundary line switches to isolate the faulted zone. The upstream service is then restored by closing the feeder circuit breaker (FCB). The unfaulted but out-of-service zones are restored by closing the open-tie switch if the supporting feeder and main transformer have enough reserve capacity. Fault detection, isolation, and restoration (FDIR) is considered to be one of the major functions of feeder automation to improve service reliability. In Taipower, the goal of FDIR is to reduce the average service restoration time from $58 \mathrm{~min}$ to less than $5 \mathrm{~min}$ for a contingency occurring on a distribution feeder. ${ }^{(1)}$

In FDIR, switching operation strategies are usually determined by the volume of realtime data. Without an effective decision support tool, distribution dispatchers can only rely on their past experience in devising operating strategies based on the real-time data they receive. A colored Petri net $(\mathrm{CPN}),{ }^{(2-7)}$ which is an extended model of Petri nets, is a graphical and mathematical modeling method with a powerful inference mechanism for describing information processing systems that are characterized as being concurrent, asynchronous, distributed, parallel, nondeterministic, or stochastic. Compared with Petri nets, the token set of a CPN, which is a vector, contains more information and represents more states of a system. Many interesting applications of CPNs in the power field have been reported, such as repair scheduling and dispatching of large-scale contingencies in distribution systems, ${ }^{(8)}$ load balancing of distribution systems, ${ }^{(9-11)}$ optimal switching operations for the service restoration and loading balancing of distribution systems, ${ }^{(12)}$ and temperature-adaptive load transfer of distribution systems. ${ }^{(13)}$

In this study, an inference mechanism of switching operations based on a CPN approach is applied to determine the strategy of service restoration. The real-time data of the DAS, including the status of FCBs and automatic switches, and overcurrent fault flags reported by FTUs are considered in the mechanism.

The remainder of this paper is organized as follows. Section 2 briefly describes the function of FDIR for feeder automation. Section 3 describes the operational rules of the feeder automation of Taipower that are used in this study. Section 4 illustrates the CPN models of FDIR. Section 5 provides simulation results. Finally, Sects. 6 and 7 respectively give a discussion and conclusions.

\section{FDIR}

To improve operational efficiency in terms of the system average interruption duration index (SAIDI) and system average interruption frequency index (SAIFI), Taipower launched the DAS as shown in Fig. 1. When a permanent fault occurs, the FCB and the overcurrent relays of all upstream FTUs are tripped. The fault flags of FTUs are transmitted to the master station of the DAS via an optical fiber network. Once the fault is located, switches on both sides of the fault are opened to isolate the faulted zone as the first step of FDIR. With the faulted service zones of the feeder isolated, the upstream service is then restored by closing the FCB. Downstream service restoration is a more complicated procedure than upstream restoration because multiple 


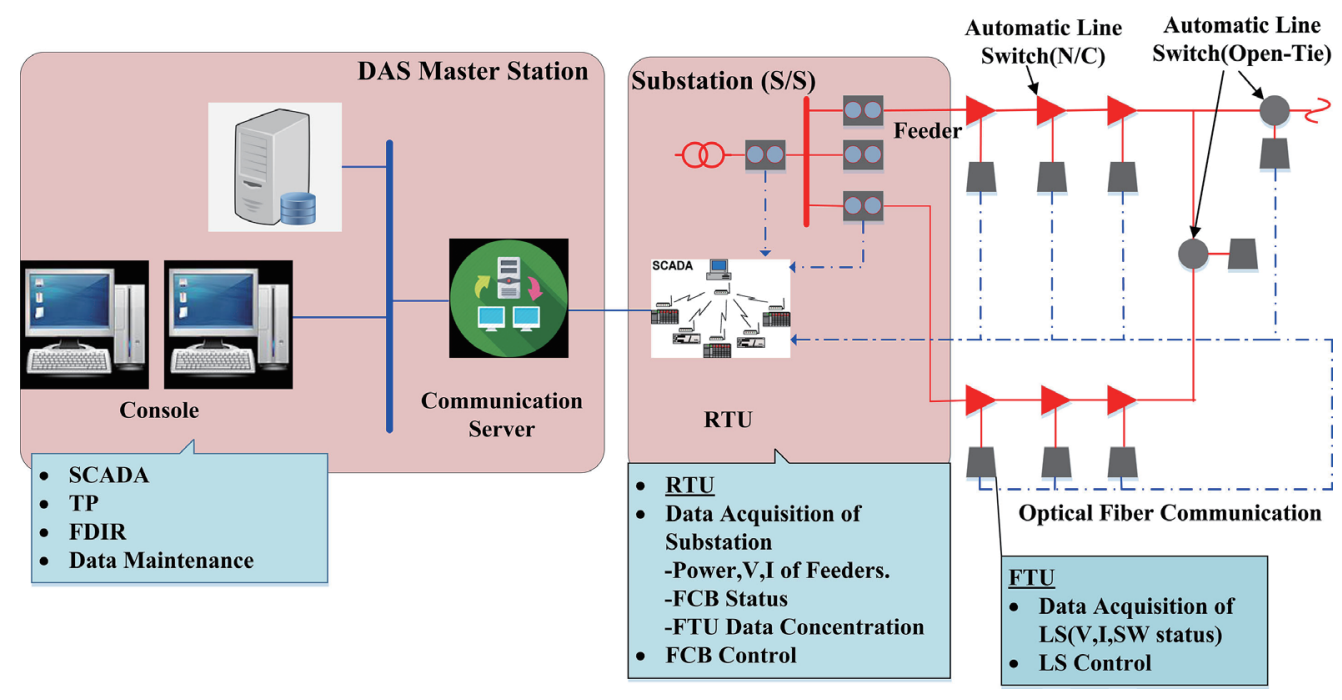

Fig. 1. (Color online) Configuration of DAS in Taipower.

sets of switching combinations should be decided by the DAS. The supporting feeder and main transformer with the largest reserve capacity are considered for load transfer before the corresponding open-tie switch is closed.

The master station consists of the supervisory control and data acquisition (SCADA) function for substations and distribution system automation, a topology process, an outage management system, and real-time data servers. ${ }^{(1)}$ The master station is implemented with a distributed client/server structure. Field data are collected from the substations via DNP3.0 over a TCP/IP LAN to the substation.

A remote terminal unit (RTU) not only monitors the parameters of the main transformers such as the statuses of circuit breakers, currents, voltages, and real/reactive power but also polls the downstream FTUs along the feeders in the substation. The data of all FTUs are collected and processed by the substation RTU and forwarded to the master station. The RTU isolates the faulted service zones, verifies the isolation, and recloses the FCB based on the feeder topology, which is downloaded from the master station. The processes of fault isolation and upstream service restoration are accomplished within $20 \mathrm{~s}$. The next $20 \mathrm{~s}$ are used to compute the reserve capacity of the supporting feeders and propose the strategy of switching operations to the distribution dispatchers for downstream service restoration.

To effectively create the CPN model and efficiently perform the FDIR procedure and postevent recovery in a distribution system, the topology process is executed to determine the energization status of automatic switches on a feeder under abnormal operating conditions. The inference mechanism of the CPN used to perform the FDIR function is based on the configuration of distribution feeders, SCADA data from the RTU and FTU, and Taipower's switching operation rules. Figure 2 illustrates a flowchart of the proposed inference mechanism of the CPN for FDIR. 


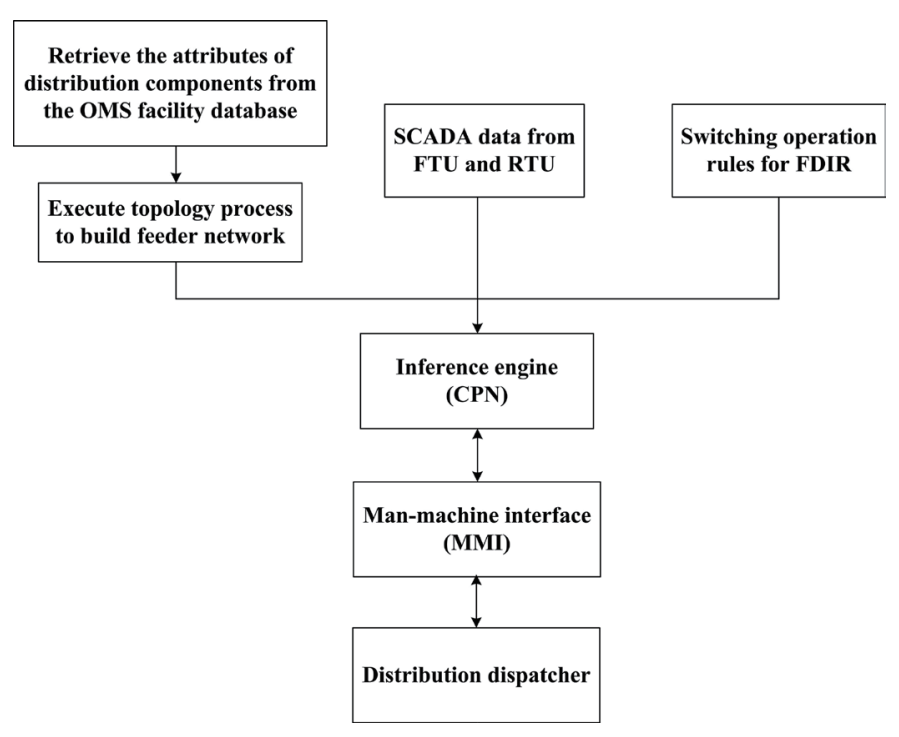

Fig. 2. Flowchart of the proposed inference mechanism of the CPN for FDIR.

\section{Operational Rules for Feeder Automation of Taipower}

The section introduces the operating rules used in Taipower's DAS. ${ }^{(14)}$

1. Restore power to most customers as soon as possible in out-of-service unfaulted areas.

2. Restore power to critical customers that provide emergency services with a high priority.

3. Minimize number of switching operations for FDIR.

4. Distribution network remains in the open-loop status after service is restored.

5. Switching strategy does not overload distribution facilities.

In this article, Taipower's operational rules for FDIR are applied to derive the switching strategy after a permanent fault is located and is isolated by automatic line switches. The operational rules should be executed before the transitions of the CPN model are enabled.

When a permanent fault occurs on a feeder, the overcurrent relay detects the fault and FTUs send fault flags to the DAS. Automatic line switches are opened to isolate the outage service zones, and the upstream service zones are then restored by closing the FCB. For the downstream restoration, the following operation rules are applied to perform the switching operation for service restoration. ${ }^{(15)}$

\subsection{Restoration for a feeder fault}

Rule1: Unfaulted but-out-of-service zones are restored by closing an open-tie switch, and the loadings of downstream service zones are then transferred to the other supporting feeder. The supporting feeder and main transformer should have enough reserve capacity to support the outage service zones.

Rule2: The DAS executes load shedding based on the service priority of customers before the interrupted load transfer is performed when the supporting main transformer has insufficient reserve capacity. 


\subsection{Restoration for a feeder outlet fault}

Rule3: The supporting feeder with the largest reserve capacity is chosen for the load transfer of the entire interrupted feeder.

Rule4: The DAS executes partial load transfer for the outage feeder when the supporting feeder has insufficient reserve capacity.

Rule5: Rule2 is applied for fault restoration when the supporting main transformer has insufficient reserve capacity.

The open-tie switch that connects the downstream service zones is considered as a potential line switch to be operated. The DAS chooses the candidate open-tie switch of the supporting feeder with the largest reserve capacity to execute load transfer. The supporting feeder in the same substation has a higher priority for interrupted load transfer to minimize the impact of outage of a distribution system.

\section{Colored-Petri-Net-Based Modeling of FDIR}

CPNs, an extension of high-level Petri nets, are applied for modeling complex systems with a dynamic process. CPNs, different from traditional Petri nets, add another dimension to tokens, which are characterized by an attached color to represent different functions. In this study, various token colors are represented as the statuses of circuit breakers and automatic line switches (two-way and four-way switches). An attached color is assigned to each token of a CPN. These different token colors can then be used to determine which transition of the CPN model can operate. The state of a CPN, i.e., a marking, consists of many tokens positioned at individual places. In the FDIR procedure, the initial marking is determined by the statuses of circuit breakers and automatic line switches. The formal definition of a CPN can be found in Ref. 15.

In this study, the dynamic property is utilized to model the processes of FDIR. The design consideration and the definitions for the CPN modeling of FDIR are based on the topology of the distribution network. Figure 3 shows a simple distribution feeder with automatic two-way

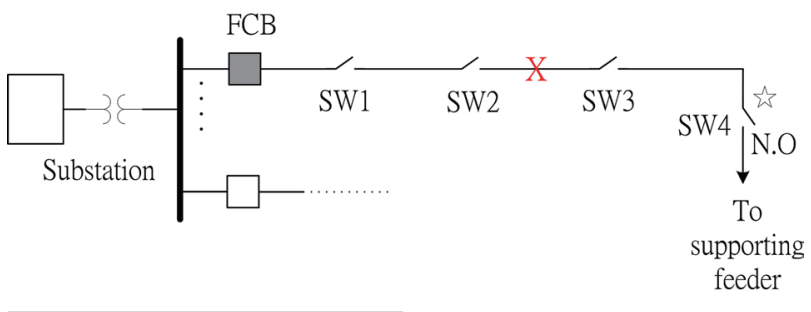

$$
\begin{aligned}
& \square: \text { feeder circuit breaker } \\
& \overline{\mathrm{N}} . \overline{\mathrm{O}} \text { : open-tie switch }
\end{aligned}
$$

Fig. 3. (Color online) Simple distribution feeder with automatic two-way line switches. 
line switches to illustrate the inference mechanism of the CPN model for FDIR. The FDIR based on the CPN model for the system shown in Fig. 3 is depicted in Fig. 4.

In Fig. 4, each place can be marked by attaching a data value (token color). In the CPN model of Fig. 4, the data values of five place nodes (P0, P1, P7, P8, and P9) for the FDIR process and five place nodes (P2, P3, P4, P5, and P6) for the circuit breaker and automatic line switches are illustrated in Tables 1 and 2, respectively. For instance, the state of place R1 (P1) indicates that the DAS performs the FDIR process of feeder automation. Four-way and

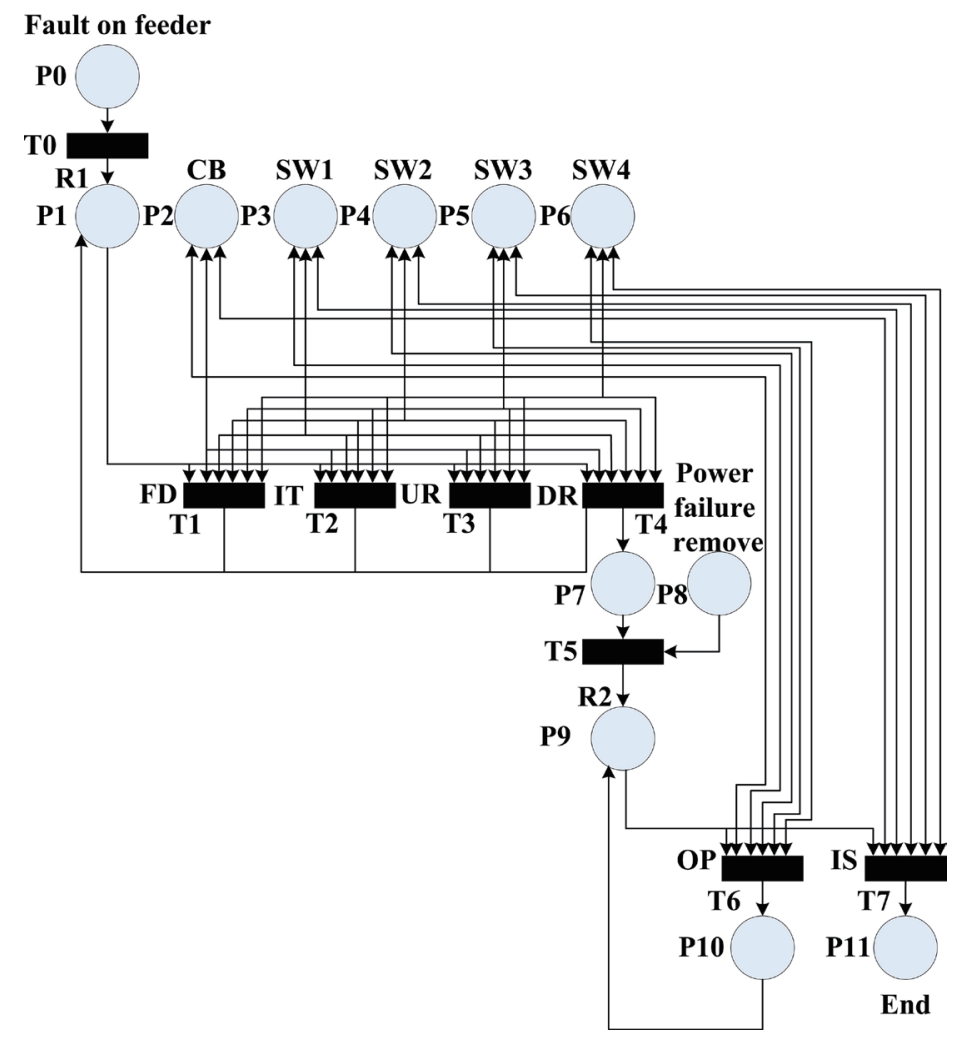

Fig. 4. (Color online) FDIR model of the test system based on a CPN.

Table 1

Place nodes for the states of the FDIR process.

\begin{tabular}{lll}
\hline Places & Token colors & Description \\
\hline P0 & P0_NF & Non-fault on a feeder \\
\cline { 2 - 3 } & P0_F & Fault on a feeder \\
\hline \multirow{2}{*}{ P1 } & P1_F & Fault on a feeder \\
\hline P1_FD & Fault detection process \\
\hline P1_FI & Fault isolation process \\
\hline P1_UR & Upstream service zone restoration \\
\hline P7 & P1_DR & Downstream service zone restoration \\
\hline P8 & P7_complete & FDIR process is completed \\
\hline P9 & P8_wt_remove & Power failure is not removed \\
\cline { 2 - 3 } & P8_remove & Power failure is removed \\
\hline
\end{tabular}


Table 2

Place nodes for the states of circuit breaker and automatic line switches.

\begin{tabular}{|c|c|c|}
\hline Places & Token colors & Description \\
\hline \multirow{2}{*}{ P2 } & P2_nf_close & $\begin{array}{l}\text { 1. } \mathrm{CB} \text { without overcurrent flag } \\
\text { 2. } \mathrm{CB} \text { is closed }\end{array}$ \\
\hline & P2_f_open & $\begin{array}{l}\text { 1. } \mathrm{CB} \text { with overcurrent flag } \\
\text { 2. } \mathrm{CB} \text { is tripped }\end{array}$ \\
\hline \multirow{3}{*}{$\begin{array}{l}\text { P3, P4, P5, P6 } \\
\text { (Two-way automatic line } \\
\text { switch) }\end{array}$} & P*_nf_close & $\begin{array}{l}\text { 1. SW without overcurrent flag } \\
\text { 2. SW is closed }\end{array}$ \\
\hline & P*_nf_open & $\begin{array}{l}\text { 1. SW without overcurrent flag } \\
\text { 2. SW is opened }\end{array}$ \\
\hline & $\mathrm{P}^{*}$ _f_close & $\begin{array}{l}\text { 1. SW with overcurrent flag } \\
\text { 2. SW is closed }\end{array}$ \\
\hline \multirow{5}{*}{$\begin{array}{l}\text { P3, P4, P5, P6 } \\
\text { (Four-way automatic line } \\
\text { switch) }\end{array}$} & $\mathrm{P}^{*}$ _nf_open_open & $\begin{array}{l}\text { 1. SW without overcurrent flag } \\
\text { 2. SW1 is opened } \\
\text { 3. SW2 is opened }\end{array}$ \\
\hline & $\mathrm{P}^{*}$ nf_open_close & $\begin{array}{l}\text { 1. SW without overcurrent flag } \\
\text { 2. SW1 is opened } \\
\text { 3. SW2 is closed }\end{array}$ \\
\hline & P*_nf_close_open & $\begin{array}{l}\text { 1. SW without overcurrent flag } \\
\text { 2. SW1 is closed } \\
\text { 3. SW2 is opened }\end{array}$ \\
\hline & P*_nf_close_close & $\begin{array}{l}\text { 1. SW without overcurrent flag } \\
\text { 2. SW1 is closed } \\
\text { 3. SW2 is closed }\end{array}$ \\
\hline & $\mathrm{P}^{*}$ _f_close_close & $\begin{array}{l}\text { 1. SW with overcurrent flag } \\
\text { 2. SW1 is closed } \\
\text { 3. SW2 is closed }\end{array}$ \\
\hline
\end{tabular}

*: place node number

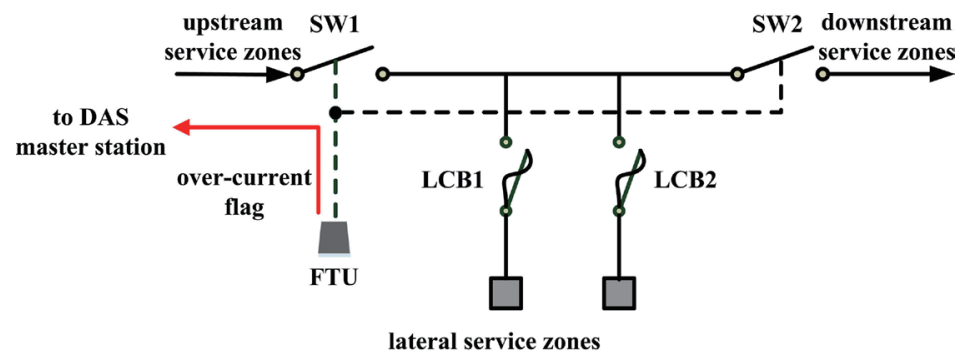

(a)

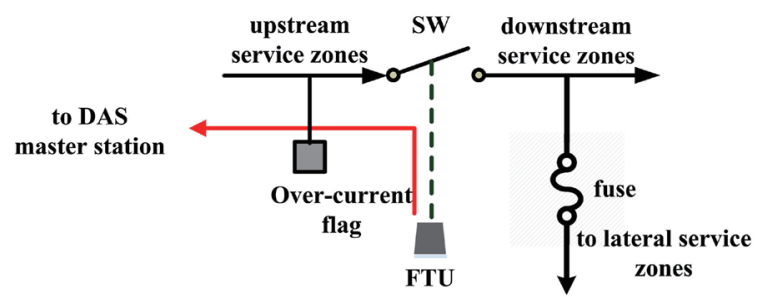

(b)

Fig. 5. (Color online) Automatic line switches: (a) four-way line switch and (b) two-way line switch.

two-way automatic line switches are respectively installed in the underground and overhead distribution systems for fault isolation and service restoration as shown in Fig. 5. For four- 
way line switches, two load break switches (LBSs) are installed to enable remote control of the connection and disconnection of the primary line sections shown in Fig. 5(a). The LBSs SW1 and SW2 are respectively located in near upstream service zones and downstream service zones to execute the fault isolation process. The command of the overcurrent flag is sent to the DAS master station when a permanent fault occurs. The five token colors for a four-way line switch consist of the status of the overcurrent flag, SW1, and SW2. The three token colors for a twoway line switch are represented as the status of the overcurrent flag and SW. The DAS performs FDIR and power recovery processes for feeder automation during a contingency. Based on Fig. 5, Table 3 illustrates the token colors of the CPN model for the FDIR and power recovery processes.

When a transition is enabled, the color tokens are removed from input places, and appropriate tokens based on the FDIR and power recovery operation rules are added to output places. The available reserve capacities of the potential supporting feeders and main transformers are evaluated on the basis of the loadings of the supporting feeders and the main transformer collected from FTUs and the RTU of the DAS before the transition nodes of the CPN model are enabled. For instance, a permanent fault occurs on the distribution feeder between switches SW2 and SW3 in Fig. 3. The reserve capacities of the supporting feeder and the supporting main transformer must be evaluated before the candidate open-tie switch SW4 closes. The transition T4 is then activated and the token color of place P6 for switch SW4 changes from P6_nf_open to P6_nf_close (Table 3). If the supporting main transformer has insufficient reserve capacity to execute the load transfer, the partial loading of the main transformer must be transferred to another feeder and then open-tie switch SW4 is closed. When the faulted feeder section is repaired, place P8 receives a token and transition T5 is enabled. The DAS performs the power recovery processes. The sequence is switched back to the pre-fault configuration when the fault is cleared. Open-tie switch SW4 closes (enabling transition T6), then boundary line switches SW2 and SW3, which isolate the faulted zone, are closed (transition T7 is enabled).

\section{Case Study}

In this section, an FDIR simulation of Taipower's distribution system is performed. The one-line diagram of the test system is illustrated in Fig. 6. The CPN model is developed in C++

Table 3

Token colors of CPN model for FDIR and power recovery processes.

\begin{tabular}{|c|c|c|c|c|c|c|c|c|}
\hline \multirow[b]{3}{*}{ Places } & \multicolumn{8}{|c|}{ Token colors } \\
\hline & \multirow[b]{2}{*}{$\begin{array}{l}\text { Normal } \\
\text { condition }\end{array}$} & \multirow[b]{2}{*}{$\begin{array}{l}\text { Fault on a } \\
\text { feeder }\end{array}$} & \multicolumn{4}{|c|}{ FDIR process } & \multicolumn{2}{|c|}{ Power recovery process } \\
\hline & & & Fault detection & Fault isolation & $\begin{array}{l}\text { Upstream } \\
\text { restoration }\end{array}$ & $\begin{array}{c}\text { Downstream } \\
\text { restoration }\end{array}$ & $\begin{array}{c}\text { Open-tie switch is } \\
\text { opened }\end{array}$ & $\begin{array}{c}\text { Boundary } \\
\text { switches are } \\
\text { closed }\end{array}$ \\
\hline P0 & P0_NF & P0_F & * & * & $*$ & * & * & $*$ \\
\hline P1 & * & P1_FD & P1_FI & P1_UR & P1_DR & $*$ & * & $*$ \\
\hline P2 & P2 nf close & P2 f open & P2 f open & P2 f open & P2 nf close & P2 nf close & P2 nf close & P2_nf_close \\
\hline P3 & P3_nf_close & P3_f_close & P3_f_close & P3_f_close & P3_f close & P3_f_close & P3_f_close & P3_f_close \\
\hline P4 & P4_nf_close & P4_f close & P4_f close & P4_f_open & P4f open & P4 f open & P4f f open & P4f c close \\
\hline P5 & P5_nf_close & P5_nf_close & P5_nf_close & P5_nf_open & P5_nf_open & P5_nf_open & P5_nf_open & P5_nf_close \\
\hline P6 & P6 nf open & P6 nf open & P6 nf open & P6 nf open & P6 nf open & P6 nf close & P6 nf open & P6 nf open \\
\hline P7 & $*$ & * & $*$ & * & * & P7_complete & $*$ & $*$ \\
\hline P8 & * & P8_wt_remove & P8_wt_remove & P8_wt_remove & P8_wt_remove & P8_remove & * & * \\
\hline P9 & * & * & * & * & * & P9_complete & P9_OPSW_open & P9_OPSW_open \\
\hline P10 & * & * & * & * & * & * & * & P10_complete \\
\hline
\end{tabular}




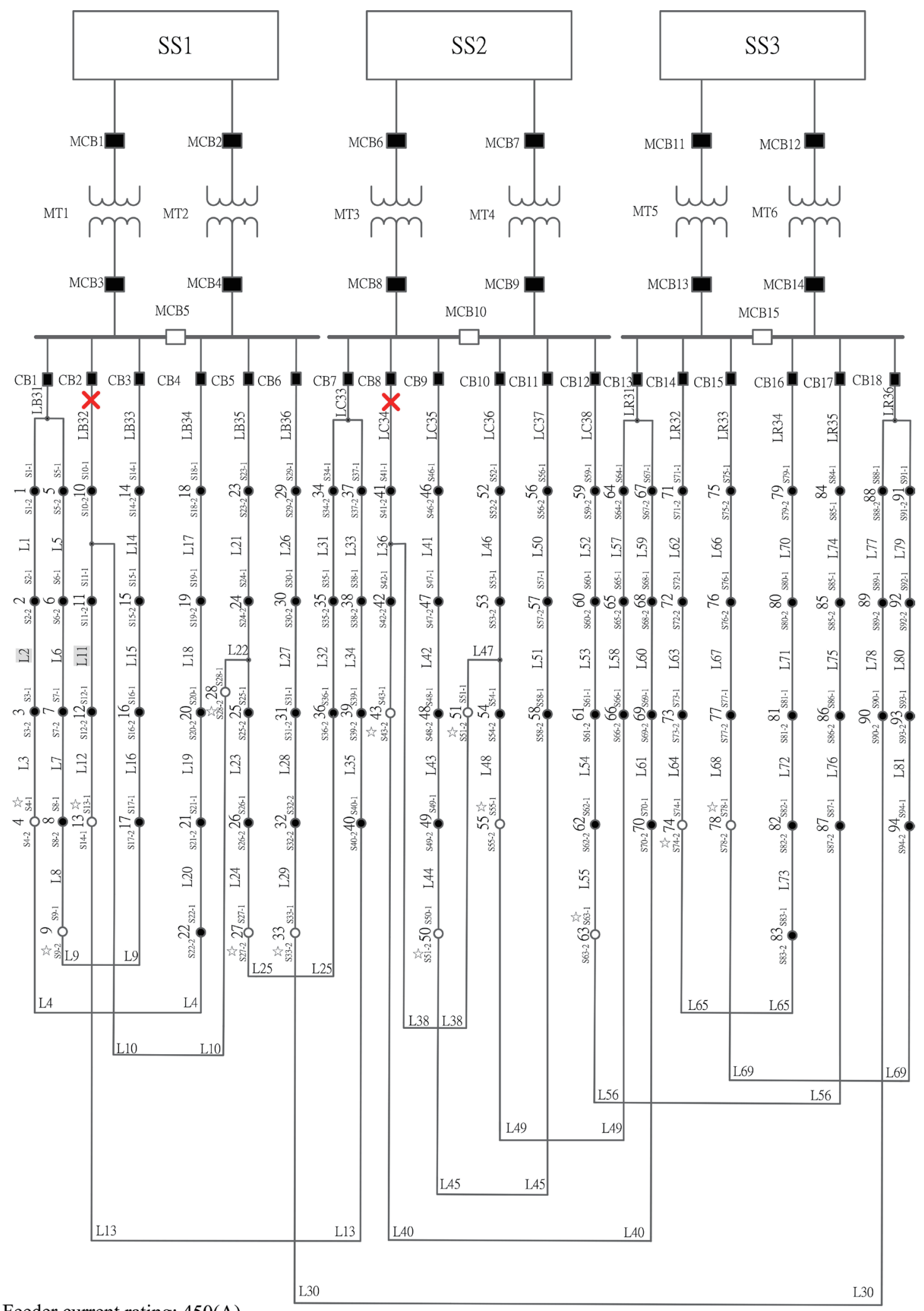

Feeder current rating: 450(A)

Transformer current rating: 604 (A)

Fig. 6. (Color online) One-line diagram of the study system. 
and run on a personal computer (Intel Core i7 $4.6 \mathrm{GHz}$ ) to perform the FDIR strategy. Reserve capacities of the main transformer that are sufficient and insufficient are chosen to simulate the switching operation of FDIR. In the following subsections, the notation (X, Y) represents the operations of opening switch $\mathrm{X}$ then closing switch $\mathrm{Y}$ to maintain the open-loop distribution network.

\subsection{Case A: Fault on feeder outlet (with enough reserve capacity to support main transformer)}

In this case, a fault occurs on the outlet of feeder LC34, and the CPN model is developed using the topology of feeder LC34 shown as Fig. 7. The fault is detected by the statuses of the circuit breaker and line switches along feeder LC34. Circuit breaker CB8 is tripped and line switch S41-1 is opened after the fault is detected. Circuit breaker CB8 is closed when the isolation process is confirmed. The DAS then performs load transfer from LC34 to two of the supporting feeders, LR31 and LC36, based on their reserve capacities for downstream service restoration. Table 4 illustrates the reserve capacity margins of the faulted feeder, supporting feeder, and main transformer. The reserve capacity of supporting feeder LC36 is larger than that of LR31, and the main transformer MT4 has enough reserve capacity to take over the loading of the faulted feeder LC34. The loading (176.5 A) of LC34 is transferred to feeder LC36 by closing switch SW41-2 for downstream restoration.

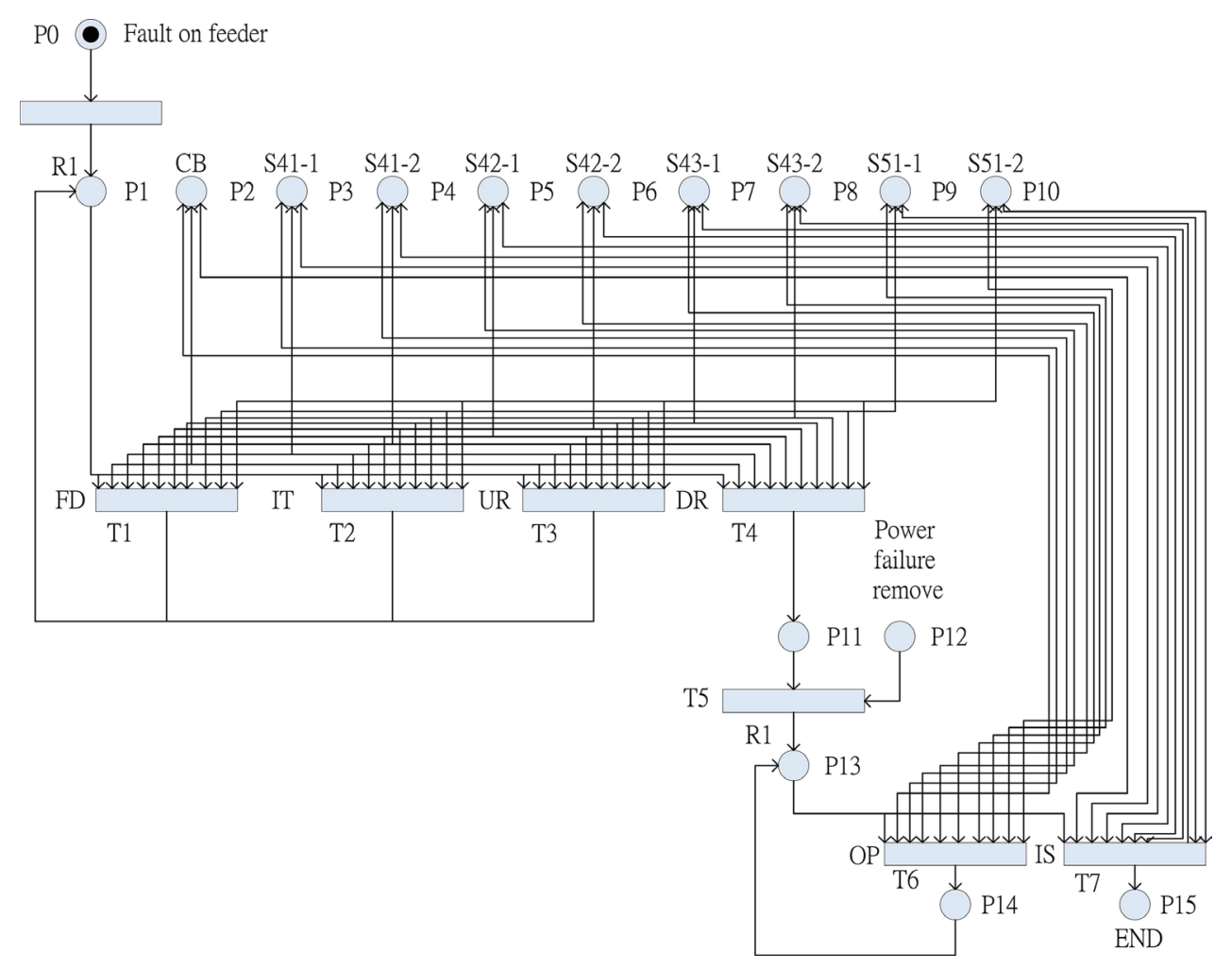

Fig. 7. (Color online) CPN model for Case A. 
Table 4

Reserve capacities of the supporting feeder and main transformer.

\begin{tabular}{ccc}
\hline Feeder/Main transformer & Load current $(\mathrm{A})$ & FRCM/TRCM $^{*}$ \\
\hline LC34 & 176.5 & 273.5 \\
LC36 & 186 & 264 \\
MT4 & 380.7 & 223.3 \\
LR31 & 223 & 227 \\
MT5 & 385.4 & 218.6 \\
\hline
\end{tabular}

*Feeder reserve capacity margin $(\mathrm{FRCM})=450 \mathrm{~A}$ load current

${ }^{*}$ Transformer reserve capacity margin $(\mathrm{TRCM})=604 \mathrm{~A}$ load current

\subsection{Case B: Fault on feeder outlet (without enough reserve capacity for main transformer)}

The same scenario as case A except with insufficient reserve capacity on MT4 is applied to the test system in Fig. 6. The loading of the main transformer MT4 is assumed to be $450 \mathrm{~A}$ and the reserve capacity margin of MT4 is reduced to $154 \mathrm{~A}$, which is not enough to support the loading of LC34 (176.5 A). To solve the problem, part of the loading of LC38 is transferred to LR35 by executing the switching operation (S60-2, S63-2) to provide more reserve capacity for MT4. After that, switch S51-2 closes to restore the power service of feeder LC34.

\subsection{Case C: Multiple line faults}

In Case C, a massive storm inflicts damage to low-lying distribution rooms and some faults occur in zone L2 of feeder LB31 and the outlet of feeder LB32 in Fig. 6. The faults are isolated by opening switches S2-2 and S3-1 for the L2 fault and opening circuit breaker CB2 for the outlet fault of feeder LB32. For the L2 fault, the unfaulted but out-of-service zone L3 is transferred from feeder LB31 to LB34. For the outlet fault of feeder LB32, the whole loading of feeder LB32 is transferred to feeder LB35. As a result of this operation, the main transformer MT2 has insufficient reserve capacity for the operation of load transfer. Therefore, part of the loading of LB36 is transferred to LR36 by executing the switching operation (S31-2, S33-2) to provide more reserve capacity for MT6. After that, the switching operations (S3-1, S4-1) are performed and switch S28-2 is closed to restore the services of L2 and feeder LB32.

\section{Discussion}

The novelty of this study is the application of the inference mechanism of switching operations based on the CPN approach to determine the strategy of service restoration. The real-time data of the status of FCBs and automatic switches, and overcurrent fault flags reported by FTUs are considered in the mechanism. To perform the FDIR function, the inference mechanism of the CPN is based on the configuration of distribution feeders, SCADA data from the RTU and FTU, and Taipower's switching operation rules. Compared with the traditional Petri net models, the proposed CPN model significantly reduces the computing time and memory required to derive suitable switching operations of FDIR. 


\section{Conclusions}

Feeder automation reduces the customer outage duration when a fault contingency occurs in a distribution feeder. In feeder automation operation, the FTU measures and monitors the voltage and current sensors of distribution line switchgear in real time. In this article, an inference mechanism based on a CPN is proposed to derive the switching operations of FDIR in a distribution system. Taipower's operation rules are incorporated into the CPN model and the inference mechanism to derive the switching strategy based on the operating regulation. The inference mechanism integrated with the operation rules is applied to find the restoration strategy for fault detection and isolation, and service restoration of distribution systems. To demonstrate the effectiveness of the proposed methodology, a Taipower distribution system with 18 feeders is investigated in a computer simulation. According to the computer simulation, the proposed methodology provides an effective tool for distribution dispatchers to execute feeder automation of a distribution system. The outage duration can be reduced and the system reliability improved by the inference mechanism based on the CPN when a fault occurs on a feeder.

\section{References}

1 G. L. Ockwell: Proc. 2003 IEEE Power Engineering Society General Meeting (IEEE, 2003) 2430-2434. https://doi.org/10.1109/PES.2003.1271022

2 C. G. Cassandras and S. Lafortune: Introduction to Discrete Event Systems (Springer-Verlag 2008) 2nd ed., Chap. 4. https://doi.org/10.1007/978-0-387-68612-7

3 B. Hruz and M. Zhou: Modeling and Control of Discrete- Event Dynamical System: with Petri Nets and Other Tool (Springer, 2007) Chap. 8. https://doi.org/10.1007/978-1-84628-877-7

4 P. Sun and C. Jiang: Inf. Software Technol. 51 (2009) 2. https://doi.org/10.1016/j.infsof.2008.02.004

5 K. Jensen, L. M. Kristensen, and L. Wells: Int. J. Software Tools Technol. Transfer 9 (2004) 3. https://doi. org/10.1007/s10009-007-0038-x

6 R. Wang and C. H. Dagli: Proc. 2008 2nd Annu. IEEE Systems Conf. (IEEE 2008) 1-8. https://doi.org/10.1109/ SYSTEMS.2008.4518997

7 R. Robidoux, H. Xu, L. Xing, and M. Zhou: IEEE Trans. Syst. Man Cybern. Part A Syst. Humans 40 (2010) 2. https://doi.org/ 10.1109/TSMCA.2009.2034837

8 T. H. Chang, T. E. Lee, and C. H. Lin: Proc. 2017 IEEE Int. Conf. Applied System Innovation (IEEE 2017) 1036-1039. https://doi.org/10.1109/ICASI.2017.7988118

9 C. H. Lin: IEE Proc. Generation, Transmission and Distribution 150 (2003) 3. https://doi.org/10.1049/ipgtd:20030199

10 Y. L. Ke, C. S. Chen, M. S. Kang, J. S. Wu, and T. E. Lee: IEEE Trans. Power Systems 19 (2004) 1. https://doi. org/10.1109/TPWRS.2003.821433

11 T. H. Chang, T. E. Lee, and C. H. Lin: Proc. 2017 IEEE Int. Conf. Applied System Innovation (IEEE 2017) 1040-1043. https://doi.org/10.1109/ICASI.2017.7988119

12 R. V. Parimala and K. L. Puttabuddhi: Proc. 2011 Int. Conf. Sustainable Energy and Intelligent Systems (SEISCON 2011) 826-832. https://doi.org/10.1049/cp.2011.0480

13 Y. C. Chuang, Y. L. Ke, C. S. Chen, and Y. L. Chen: IEEE Trans. Power Systems 21 (2006) 3. https://doi. org/10.1109/TPWRS.2006.879309

14 C. S. Chen, C. H. Lin, and H. Y. Tsai: IEEE Trans. Power System 17 (2002) 4. https://doi.org/10.1109/ TPWRS.2002.804947

15 K. Jensen and L. M. Kristensen: Coloured Petri Nets-Modelling and Validation of Concurrent Systems (Springer-Verlag, 2009) Chap. 4. https://doi.org/10.1007/b95112 


\section{About the Authors}
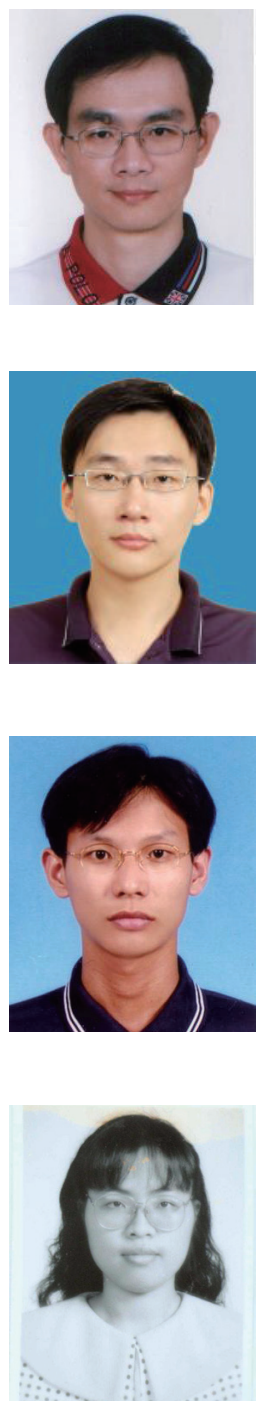

Chia-Hung Lin received his B.S. degree from National Taiwan Institute of Technology, Taiwan, in 1991 and his M.S. and Ph.D. degrees from University of Pittsburgh, USA, in 1993 and from University of Texas at Arlington, USA, in 1997, respectively. Since 2001, he has been a professor at National Kaohsiung University of Science and Technology. His research interests are in smart grids. (chlin@nkust.edu.tw)

Chung-Sheng Li received his B.S. and M.S. degrees from National Kaohsiung University of Science and Technology, Taiwan, in 2003 and 2005, respectively. His research interests are in smart grids. (nargal@gmail.com)

Te-Tien Ku received his M.S. and Ph.D. degrees from National Sun YatSen University, Taiwan, in 2006 and 2012, respectively. Since 2017, he has been an associate professor at National Kaohsiung University of Science and Technology. His research interests are in smart grids and applications to distribution systems. (ttku@nkust.edu.tw)

Chin-Ying Ho received her Ph.D. degree from National Kaohsiung University of Applied Sciences, Taiwan, in 2010. Since 2010, she has been an associate professor at Kao Yuan University. Her research interests are in the areas of load surveys and computer applications to power systems.

(tf0078@cc.kyu.edu.tw) 